\title{
Lightning Talk: Biopython (bio) Geography Module
}

\section{Nicholas J. Matzke \\ matzke@berkeley.edu \\ Department of Integrative Biology \\ University of California, Berkeley}

\begin{abstract}
For Google Summer of Code 2009/NESCENT Phyloinformatics Summer of Code 2009, I built a Geography module for Biopython. The purpose of the module is to search, download, and process biogeographical data from GBIF, much as Biopython currently accesses Genbank. Application of the tool to a historical biogeography study on bivalves will be illustrated.

As required by Google Summer of Code and Biopython, the code is open access and is released under the Biopython License:

http://www.biopython.org/DIST/LICENSE
\end{abstract}

The module is described, and a tutorial is presented, on the Biopython wiki:

http://biopython.org/wiki/BioGeography

The page contains links to the source hosted on Github; here is the direct link:

http://github.com/nmatzke/biopython/tree/Geography 